\title{
John A. LYNN II, Women, Armies, and Warfare in Early
}

\section{Modern Europe}

New York, Cambridge University Press, 2008, 239 pages

\section{Marion Trévisi}

\section{CpenEdition}

Journals

\section{Édition électronique}

URL : http://journals.openedition.org/clio/9731

DOI : $10.4000 /$ clio. 9731

ISSN : 1777-5299

Éditeur

Belin

Édition imprimée

Date de publication : 1 mai 2010

ISSN : 1252-7017

Référence électronique

Marion Trévisi, « John A. Lynn II, Women, Armies, and Warfare in Early Modern Europe ", Clio. Femmes,

Genre, Histoire [En ligne], 31 | 2010, mis en ligne le 17 juin 2010, consulté le 21 septembre 2020. URL

http://journals.openedition.org/clio/9731 ; DOI : https://doi.org/10.4000/clio.9731

Ce document a été généré automatiquement le 21 septembre 2020.

Tous droits réservés 


\section{John A. LYNN II, Women, Armies, and Warfare in Early Modern Europe}

New York, Cambridge University Press, 2008, 239 pages

\section{Marion Trévisi}

1 Ce livre de synthèse sur la présence et le rôle des femmes dans les armées européennes de l'époque moderne est écrit par un spécialiste d'histoire militaire qui s'intéresse surtout aux femmes de camp qui suivaient les armées dans leurs déplacements, et moins aux femmes combattantes dont les représentations fourmillent dans les chansons, gravures et romans des trois siècles modernes. Il est vrai que les femmes de camp sont bien moins connues car, comme l'auteur le souligne dès l'introduction, les sources sont rares ou lacunaires à leur sujet; les armées ne tiennent pas de listes pour ces femmes et les administrations ne les évoquent que pour s'en plaindre (en les assimilant toutes à des prostituées) et les rejeter par des ordonnances répressives ou lors de procès militaires. John A. Lynn s'appuie ainsi sur un grand nombre de gravures de l'époque (gravures majoritairement hollandaises et allemandes) ainsi que sur des mémoires et correspondances d'officiers et de soldats de toute l'Europe pour cerner leur importance au sein des armées du début de l'époque moderne. Il soutient en effet que ces femmes de camp étaient vitales pour les troupes avant 1650 en raison des lacunes logistiques d'armées de contrat et de mercenaires et qu'elles étaient moins nombreuses après 1650 , car moins nécessaires dans des armées mieux payées, à la logistique assurée par des États plus puissants et mieux organisés. C'est ce qu'il précise dans un premier chapitre rappelant l'évolution du contexte militaire de 1500 à 1800 ; avant 1650 , les soldats sont des mercenaires sous contrat mal payés, qui pratiquent le pillage systématique pour compenser des soldes trop ponctuelles. Les femmes de camp jouent alors un grand rôle dans cette économie de pillage ainsi que dans la vie de la troupe ou de la communauté de campagne (soins, travaux et services divers). Après 1650, les États monarchiques européens disciplinent leurs armées sur le modèle de celle de Louis XIV : les soldes plus régulières, l'amélioration de la logistique (nourriture et soins), le recrutement de soldats nationaux tendent à un meilleur contrôle des troupes par les États, à un rejet du pillage, beaucoup moins bien toléré par les officiers, et donc 
au rejet des femmes de troupe, moins utiles aux soldats et mieux encadrées par l'administration. Sans oublier l'impact des réformes religieuses qui exigent une moralité plus stricte des soldats et donc l'exclusion des prostituées suivant les armées (du moins en théorie). John A. Lynn termine cette présentation générale en insistant sur l'importance des femmes de camp et de leurs activités qui auraient influencé le cours des guerres, leur conduite par les États et les réformes militaires qui ont suivi au $\mathrm{XVII}{ }^{\mathrm{e}}$ siècle. Malheureusement, cette conclusion ne se fonde sur aucune archive réelle, car les États n'ont jamais mesuré ni reconnu l'importance de ces femmes dans leur organisation militaire.

2 L'auteur présente ensuite dans le deuxième chapitre un portrait exhaustif de ces femmes de camp : des prostituées classiques assez bien tolérées au XVI ${ }^{e}$ siècle avant d'être rejetées dès le XVII ${ }^{e}$ siècle, en passant par les concubines de soldats connues sous le vocable générique de "putains » qui offraient en plus des services sexuels une aide pour le linge, la nourriture et les soins médicaux, jusqu'aux épouses légitimes des soldats, plus ou moins bien acceptées selon les États; il souligne que toutes ces femmes vivaient dans un climat de violence dû à la guerre mais aussi aux comportements des hommes de troupe vis-à-vis d'elles. Mais ces femmes fortes et courageuses participaient aussi à cette violence; elles provoquaient des bagarres entre soldats se battant pour leurs faveurs, se querellaient entre elles pour des questions de hiérarchie et de préséance (entre concubines et femmes mariées) et se battaient parfois avec des soldats pilleurs et voleurs.

3 Après ce chapitre très descriptif, c'est la vie quotidienne de ces femmes au sein des camps et leur rôle dans l'économie du pillage que retrace le troisième chapitre, le plus novateur de cette synthèse. L'auteur commence par rappeler les tâches traditionnelles des femmes dans les armées, tâches sexuées ou "genrées " que les hommes refusaient de faire afin de ne pas écorner leur masculinité : tous les travaux liés au linge et aux vêtements (laver, recoudre et habiller), à la cuisine et aux premiers soins médicaux. Cependant, les femmes de troupe devaient aussi s'atteler à des travaux plus lourds et difficiles : porter « comme des mules» les affaires des soldats (habits, sacs, nourriture, argent et parfois armes) ou encore creuser des tranchées autour du camp. Parallèlement à ces tâches, la plupart avaient des activités dans le commerce et la vente de nourriture, d'alcool et d'objets; il s'agit notamment des fameuses vivandières revendant des marchandises issues du pillage. John A. Lynn rappelle leur importance comme partenaires commerciaux et tente de détailler leur rôle exact dans l'économie du pillage: certaines suivaient leurs maris dans la mise à sac des maisons ou des champs, d'autres y allaient seules et n'hésitaient pas à se battre avec des soldats pour établir leur commerce. Même si les sources sont rares sur ce sujet (quelques mémoires), il nous semble évident que le rôle des femmes dépasse ici les frontières du genre et que ce thème nécessiterait d'être creusé par des recherches futures.

4 John A. Lynn termine son ouvrage par un chapitre sur les femmes soldats et leur importance dans la culture populaire européenne, notamment dans la littérature, les arts et les chansons où leurs exploits prennent une couleur romantique totalement fausse par rapport à la triste réalité de ces femmes qui s'engageaient le plus souvent pour fuir la misère. Il reprend ici les rares exemples connus de combattantes aux PaysBas ou en France, notamment pendant la période révolutionnaire.

5 L'intérêt de cette synthèse réside ainsi dans le large panorama des femmes de camp des armées européennes de l'époque moderne, vues à travers le prisme des 
représentations, de la littérature populaire, des mémoires et des correspondances. On pourrait regretter cependant le peu de références aux archives manuscrites des armées ou des administrations militaires. De même, le déclin du nombre des femmes de camp dans les armées européennes après 1650, qui participerait selon l'auteur à la « révolution militaire » de la seconde moitié du XVII ${ }^{\mathrm{e}}$ siècle, ne peut être chiffré avec précision et n'entraîne pas forcément de réelle "exclusion" des femmes des campagnes militaires, comme nous le savons par l'exemple des troupes napoléoniennes. Il s'agirait plus, selon nous, d'un changement de statut et de rôle pour ces femmes qui continuent de suivre les armées européennes après 1650 . 